\title{
Multi-Scale Fish Segmentation Refinement Using Contour Based Segmentation
}

\author{
Sai Ramesh $\mathrm{L}^{\mathrm{a}, 1}$, Rangapriya $\mathrm{CN}^{\mathrm{b}}$, Archana $\mathrm{M}^{\mathrm{c}}$ and Sabena $\mathrm{S}^{\mathrm{d}}$ \\ ${ }^{a}$ Teaching Fellow, Department of IST, Anna University, Tamil Nadu \\ ${ }^{\mathrm{b}}$ PG Student, Department of IST, Anna University, Tamil Nadu \\ ${ }^{\mathrm{c}}$ Lecturer, Department of Information Technology, Annamalai University, \\ Chidambaram \\ ${ }^{\mathrm{d}}$ Assistant Professor, Department of Computer Science and Engineering, Anna \\ University - Regional Centre, Tirunelveli, Tamilnadu
}

\begin{abstract}
Image processing and the analysis techniques are the increasing attention when they have enabled the non-extractive and the non-lethal approach for the collection of fisheries data. The data collection includes the following requirements such as fish size, catch estimation, regulatory compliance, species recognition and population counting. The main process that is used to measure the size of fish accurately is image segmentation. The challenges that can affect the segmentation of images include the blurring of the image areas due to the water droplets on the camera lens and the fish bodies which are out of the camera view. This project describes the automatic segmentation of fish for underwater images This segmentation algorithm implemented for identify the shape of the fish contour-based segmentation is implemented in this project. The project describes about the issues with an effective contour-based segmentation from an initial segmentation. The refinement is processed from coarse level to fine level. At the coarse level, the entire fish is aligned for the contour of the initial segmentation with trained representative contours by using iteratively reweighted least squares (IRLS). At finer levels, the refinement of contour segments is done to represent poorly segmented or missing shape parts. This method addresses the problems listed above and generates promising results with highly robust segmentation performance and length measurement.
\end{abstract}

Keywords. Contour Plot, Image Processing, Image Segmentation.

\section{Introduction}

The tracking up of the fishes and listing the total count of fishes that are observed from the dataset collected from the underwater systems. The operation is done based on the tracking up of the frames by the various detection method. The main objective of this framework is to detect the number of wishes by using online detector systems. Even though the use of online detectors is used there might be some changes that can occur

\footnotetext{
${ }^{1}$ SaiRamesh L, Department of IST, CEG Campus, Anna University, Chennai, India.

E-mail: sairamesh.ist@gmail.com.
} 
from outside the field of view. Hence in order to observe the new objects that occur randomly within the field of view a predefined tracking system has to be detected. Hence two important challenges are needed to be improved namely the tracking system and the calculation system. One is that how the high level of falsehoods and false positives are reduced due to the unreliable detection. The changes in the fish diversity, the color of fishes and the background of the fish data plays a major role in tracking up of the fishes which causes the errors while determining the tracking the fishes.

The ability to use automated image processing systems in fishing environments has attracted the attention of both the industrial and marine sciences. Counting, measuring and classifications of catches is often done directly on fishing vessels. The offline process takes much time to finish the task and also it won't be more efficient for the collection of data in research field. The automatic fish monitoring system can classify the body of the fish by measuring the length. Therefore, the development of classification standards and measuring techniques is useful and will accelerate this critical process in fishing stream. Hence the image processing techniques can help in categorization of fishes in marine regions. This was the major advantage in using image segmentation process [1]. Some of the difficulties that are faced during the Chute camera demonstrations are

1. There may be the drastic disasters and therefore there will be limitation in visibility.

2. There may be the water droplets occurred in the camera which can cause reduction in capturing clear images.

3. The body parts of the fish are not captured using infrared sensor when the view of camera is moved.

The parts of the fish's body especially tail part is not appropriate on the surface of the chute. Hence it is necessary to improve the method of classifying the strong parts and the method of measuring the fish bodies.

\section{Related works}

This gives the overview of literature surveys. It represents some of the relevant work done by the researchers. Many existing techniques have been studied by the researchers on trust evaluation; few of them are discussed below

The vision of computers has major importance on the super pixels. It is unnecessary to keep the perfect super pixel algorithms. Alippi et al. [2] compared five parts of super pixel to identify the image capabilities, memory and the performance of the section. Networks is the method to convert the most powerful visual methods. It has shown convolutional network train by pixel to pixel, providing semantic phase art. The main aim of Huang et al. [3] is to take the large size of the input images and giving back the correct size of the images in return. The main aim of Shelhamer et al. [4] is to focus on the global features in the image data instead of focusing the local images. It deals with the graph partition problem and develop it as a global technique by using image segmentation.

Chuang et al. [5] developed a new method to find the comparative segmentation of the dimensional images. The pixels are represented in the form of objects or backgrounds to produce the barriers of segmentation. Long et al. [6] proposed two main problems in the visual computer namely contour detection and the image segmentation. It states the 
algorithms of both the problems. The contour detector is the combination of number of local cues and the global framework depending upon the clustering. similarly [7] applied classification method based on image histogram segmentation which divides the entire pixel into a region having similar properties according to the gradation level. Each area after separation will match the real object, and each area will have the same internal properties, and this property is different from the surrounding area. Kanchev et al. [8] Implemented the model for the image dataset with the local shape variations in the images. This project involves the lighting and shading of the images with the use of illuminating inter image. Zivkovic et al. [9] taken the eco-friendly region as the source for the analyzation. In the year of 2009 the rules and the factors indicate the performance of both physical and chemical index of water including $\mathrm{pH}, \mathrm{DO}$ etc. The results are obtained with the standard and the high range of values for TN and COD.

Mathiassena et al. [10] The behaviour of the fish mainly depends upon the water level and its quality. Hence the environment should be monitored frequently to check the cleanliness of the resources. The relation between the mirror and the camera is to calculate the routine basis of the fish behaviour. This method takes three fish for observation. Water quality bio-monitoring utilizes close links between living organisms and the aquatic environment in which aquatic organisms rely on to complete monitoring [11]. Based on the analysis of the relationship between aquatic organisms and water quality environment. The behavior of the fewer number of fish populations are very difficult to measure correctly. To get the accurate measurements [12], a cheap price vision- based system should be built to analyze the behavior of fish in aquatic tanks. Radhakrishna Achanta et al. [13] The drinking water is the important factor for the survival of the human resources. But there is no proper purified water available in most of the regions. Considering the drinking water quality issue certain methodologies has been developed for taking the toxic tests with accuracy. Mehdi Ravanbakhsh et al. [14] done the measurement of fishes using underwater stereo video systems. But somehow the performance of the stereo video measurement has been limited due to the operational system.

Erik Rodner et al. [15] This method depends upon the convolutional neural networks that is done on the objects in order to detect and also for the classification of species. Here the removal of background is proposed which are filtered using the binary SVM classifier for the classification of fishes. Marc Chaumont et al. [16] presented two supervised machine learning methods which detects and recognizes the coral fishes in automated manner by using the underwater HD videos. Imaging technology applications plays a vital role to participate in many important areas in research and industry. These includes the process of optimization, automatic filtering and capture, and automation sorting and so on [17]. Meng-Che Chuang et al. [18] There is no limitation in the abundance of fish with the help of visual analysis that has been attracted for increasing attention. Unstable lighting, ubiquitous noise, and low frame rate (LFR) video capture in the underwater world produces the standard tracking methods that are unreliable. Kresimir Williams et al. [19] mainly shows an automatic fish segmentation algorithm to obtain the trawl based underwater camera system. The main problem that is obtained while capturing is very low brightness between the fishes and the dynamic change in the underwater background. 


\section{System Design}

\subsection{Software Description}

The execution stage regarding the task is that the complete aim is essentially changed keen on running code. Intended regarding the stage is towards interpreting the aim keen on a finest likely result within an appropriate programming language. In this section, it covers up the execution phase concerning the task, providing particulars regarding the programming language as well as improvement background employed. It as well provides a general idea about the important sections regarding the task by means of its bit by bit course. The execution phase involves the following tasks:

1. Cautious scheduling.

2. Examination regarding structure as well as constraints.

3. Aim concerning the techniques towards accomplishing the conversion.

4. Assessment concerning the conversion technique.

5. Accurate judgment about the choosing of the proposal.

6. Suitable choosing regarding the language intended for function growth.

\subsection{Design Methodologies}

Globally, there is an increasing emphasis on enhancing the efficiency of traffic management systems. Road safety, congestion control and security are the three important factors being considered, while regulating the traffic system. In the existing system, a new entity centric trust framework using decision tree classification and artificial neural networks. The proposed entity centric trust model, uses a versatile new direct and recommended trust evaluation strategy to compute trust values. In the proposed system, an efficient model that uses the self-trained network. In the proposed system, design of trust evaluation is implemented using the deep neural network model as part of improving the proposed trust evaluation better. Preprocessing the raw IOT data uses linear discriminant analysis (LDA).The neural network utilizes the self-organized mapping model with that decision making algorithm produces accurate trust evaluation

\subsection{Proposed System}

This proposed system doesn't require a greater number of datasets for training. Apart from neural network method, this proposed method gives the advantage on the data mainly focus on the fish shape. Thus, only fewer samples are required for the shape modelling. The process of removing the impurities is done mainly on the blurred segmentations. The refinement process is done and any of the segmented image taken as the input is displayed. The image taken as input does not showed in the complete accurate form. The inaccuracy is mainly due to the water droplets and the changes occurred due to lighting and also the rough contour is obtained. The refinement has been conducted based on any of the coarse to fine based system on three levels. The coarse level of each model has the alignment of the basic segmented mask whereas the local features are developed at the fine level. The segmentation of the image carries out the refinement when the region of fish is placed outside the view of camera. The usage of this method helps to recover the shape of fish easily. 


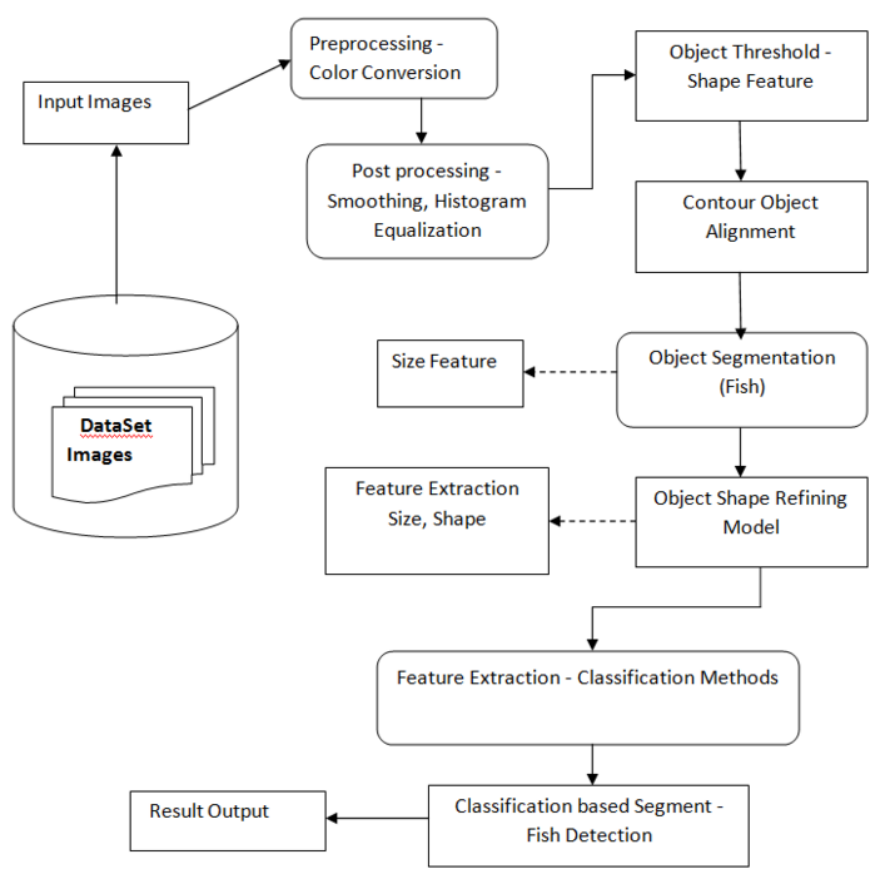

Figure 1. Architecture diagram

\subsection{Existing System}

The traditional system that contains the manual measurement consisting of the build specimens for the development of fish, monitoring and capturing the lively images of the fishes being observed and processed.

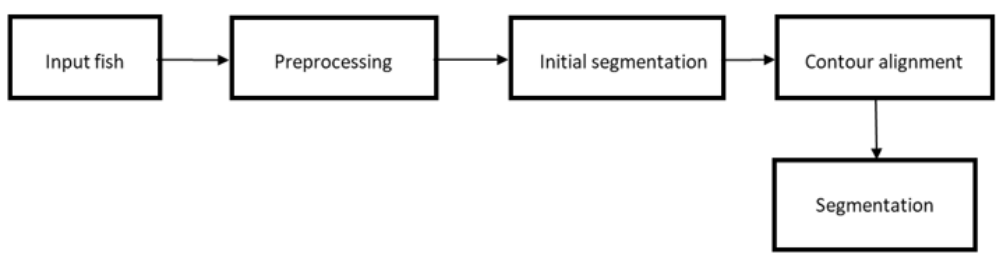

Figure 2. Dataflow diagram

\section{Implementation Methodology}

\subsection{Input Image}

The input image is read or the data is obtained from the specified file location, has specified name that changes the file format based on the contents. The file name given contains multiple number of images the function imread is used to retrieve the very first image from the file based on the size of the given image. To change or modify the size of the image in pixels, the values can be added to change the shape of given data set. 


\subsection{Image Aquitition}

The proposed system is performed by taking a recorded video and then converting the video into a number of images in order to perform the segmentation. The images that are obtained from the video is in JPG format. The high-resolution camera is mainly used for the fish ID based system. The first example is the design of development of software, collection of data and the testing of field is used in the infrared camera is used. The resolution of the RGB images permit the fishes for the easy separation from its background. The red plate is used in order to enhance the color contrast. The first system that was brought up to the development was the detection and tracking.

\subsection{Preprocessing}

At the beginning stage, the number of contour point is drawn to address the appearance of the fish. To implement this method, a contour-tracing which are related to the Pavlidis are used in a binary input image. This algorithm removes "holes" in that case that provide a sequential order of mountain pixel values. This type of tracking algorithm system is used in very easy and efficient manner for the usage of the pixel selection table in current pixel area to test and decide how it should be tested for tracking. In this second step, the points evenly spread with the extraction of curves in the form of contour pixel. This type of category is necessary to arrange points are equally arranged between two points.

\subsection{Initial Segmentation}

Obviously, the movement of the circuit from the current place in the picture, otherwise or resize it couldn't disturb the status analysis. As status of centroid is inconsistent change, rotate and measure, using solving location of translation problem. In a quiet way, the object curve is moved to location of the centroid on the links $(0,0)$. Scale increases are usually found. This type of accessible translation and measurement can be obtained by the use of regular times. In the program presented all the lower order the necessary times for body composition taken from the boundary points. This method is very fast comparison with the calculation times from the mean measurement. The same method for detection of the location $(\mathrm{m} 00)$ of the status with the simple code of chain method is used here. The route is developed at intervals of $\mathrm{m} 01$ and $\mathrm{m} 10$. The regular moment $\mathrm{mpq}$ is explained as

$$
m_{p q}=\iint x^{p} y^{q} f(x, y) d x d y
$$

The algorithm goes from one side of the point to the other side of the clock of the mount. The times are obtained with the additional method of the correct times of the trapezoids is produced from the both the position of the contour and with the $\mathrm{x}$ axis. The trapezoid has the vertices of $(x(c), y(c)),(x(p), y(p)),(x(p), 0)$ and $(x(c), 0)$ where $c$ and $p$ develop the present and past location of a line. From the above equation time formulae for lower trapezoid t order which is the function of the vertices can be found

$$
m_{00}^{t}=\frac{1}{2}[x(p)-x(c)][y(c)+y(p)]
$$




$$
\begin{aligned}
& m_{10}^{t}=\frac{1}{4}[x(p)-x(c)][x(c)+x(P)][y(c)+y(p)] \\
& m_{01}^{t}=\frac{1}{4}[y(c)-y(p)][x(c)+x(P)][y(c)+y(p)]
\end{aligned}
$$

Note that $\mathrm{mt} 00$ and $\mathrm{mt} 10$ have negative values of vertices from the upper boundary of the state (subtraction element) and positive values of vertices from the lower boundary (partial addition). Similarly, mt 01 has negative values on the left side of the shape and positive values on the right part. The method of lower order times of all shapes is obtained with the sum of the times of trapezoids

$$
m_{p q}=\sum_{\forall t \varepsilon v} m_{p q}^{t}
$$

when $\mathrm{V}$ defines all of the vertical vertices. Finally, the translation and measurement of line frequency is brought by change of the contour points of the contour according to the following variation:

$$
x^{\prime}(s)=\frac{x(s)-x_{c}}{\alpha} y^{\prime} s=\frac{y(s)-y_{c}}{\alpha}
$$

where $[\mathrm{xc}, \mathrm{yc}]$ defines a centroid connection calculated as:

$$
x_{c}=\frac{m_{10}}{m_{00}} y_{c}=\frac{m_{01}}{m_{00}}
$$

and $\alpha$ means a measurement object defined as:

$$
\alpha=\sqrt{\frac{m_{00}}{A R E A}}
$$

where AREA remains selected - the same in all cases by comparison. The rest of this project, use of $(\mathrm{x}(\mathrm{s}), \mathrm{y}(\mathrm{s}))$ in order to define the translation and measure the standard line.

\subsection{Contour Allignment}

Altering the position of an object or changing a point where it is found to be following a regional boundary should not affect the analysis of the situation. In order to avoid a test of the variability of all possible points of the first point and to maintain the strength of the defect standing it is suggested to remove a small set of points that may begin to form each point. The similarity / contrast ratio is only checked by two of the first selected locations. The accuracy and speed of searching for the right turn- based game is of the initial points selected. The method used to take the first points and the process is done for pairing the first points of the difference test can be adjusted in the given application. In this project, it was proposed a follow-up plan to remove the original location sets. 


\subsection{Segmentation}

CLAHE is operated at all of smaller locations of the image, known as tiles, rather than taking the whole image. The adapthisteq is used to calculate the contrast transformed function for every tile independently. Each one of the tiles which is contrasted is in form of enhancement. The histogram of the given output region will more or less matches the histogram mentioned by the 'Distribution' value. The nearest tiles are together formed by the use of bilinear interpolation that eliminates externally induced boundaries. The contrast, that is in homogeneous areas, might be reduce to minimize the amplification of the noise which may or may not present in the image.

\section{Simulation Results \& Discussions}

\subsection{Video Frames}

This process takes the video frames in terms of *mp4 and is get converted into number of frames. The numbers of frames are obtained to be in the form of images. The images can be given as jpg. From the given images the very first data collected from the video frame is taken as the input image and is used for further process. The image is taken from the folder where the number of video frames are stored. It is loaded by converting the video frame into image. The size can be changed in the form of pixels.

\subsection{Preprocessing}

The input image is being processed by converting the original RGB image into the Grey scale image. The image is being enhanced by using the Adaptive Histogram Equalization technique. It is the type of technique which involves the quality of the image that is given as the input that identifies the object present in an image. The Figure 3 represents the improvement of the quality of the image which enhances the entire image and calculates the contrast transform for each tile individually. The main purpose of this algorithm is to combine the neighboring tiles by using bilinear interpolation to eliminate artificial boundaries.

\subsection{Color To Grey Conversion}

The grey scale image is the one which each one of the pixels is represented using the amount of light alone. It is different from every single black and white image in terms of computer imaging. The main purpose of the conversion of color to grey image is that to reduce the total time taken for the performance of the image. The RGB image takes more time when compared to the grey scale image. A grayscale image, represented in the Figure 4 is a data matrix which the values are represented in the intensities of one image pixel. While grayscale images are rarely saved with a color map, MATLAB uses a color map to display them. 


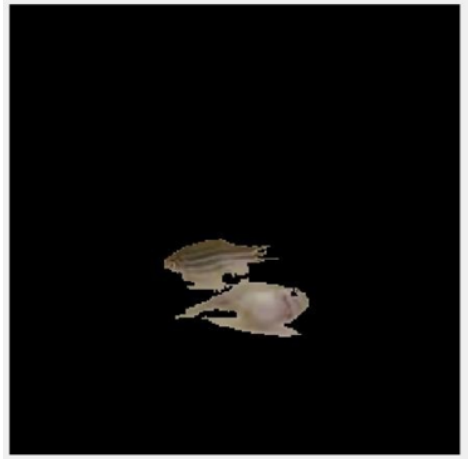

Figure 3. Input image

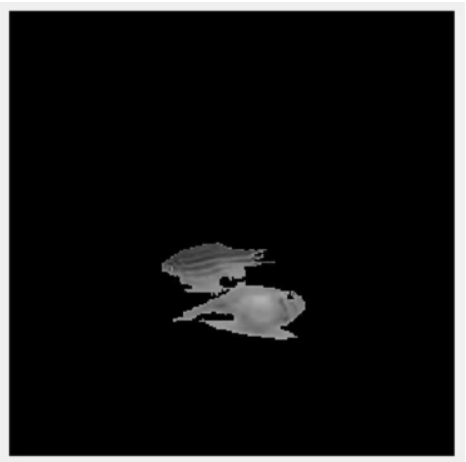

Figure 4. Grey scale Image

\subsubsection{Histogram}

Histograms is the type of bar plot for numeric data that is represented in the Figure 5 is data into bins. This can identify the changes of the image that is loaded and the output that is taken. This is used for quick modification of the properties of the bins or changing the display.

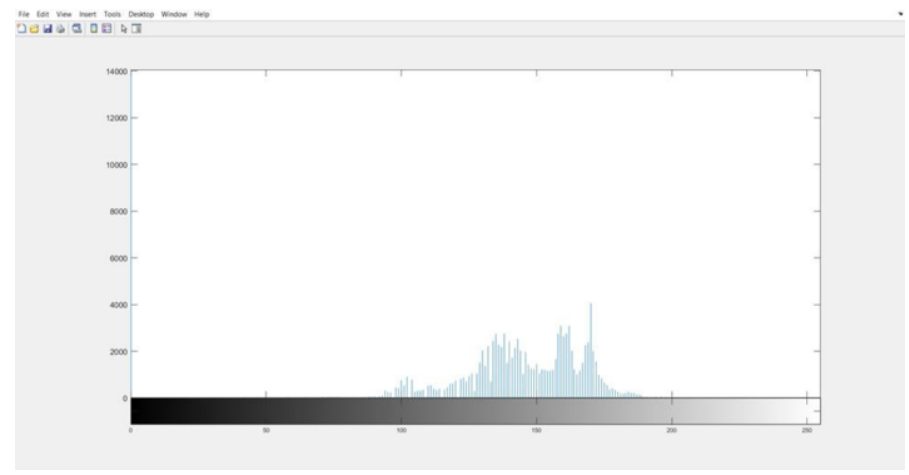

Figure 5. Histogram of grey scale image

\subsection{Contour Plot}

Contour plot represented in Figure 6 is the set of $x$ and $y$ values which indicates the $2 d$ image of given input frame. The values are given in Table 1 . The third column represents the depth of the image which recognizes the object representation in the following plain surface.

\subsection{Image Segmentation}

Image segmentation is a technique which is used in the digital image processing and analysis in order to partition each one of the images into multiple parts or regions, that is based on the characteristics of the pixels in the image. Image segmentation could involve the separation of the foreground from the background, or clustering regions of pixels that is similar to the color or shape. 


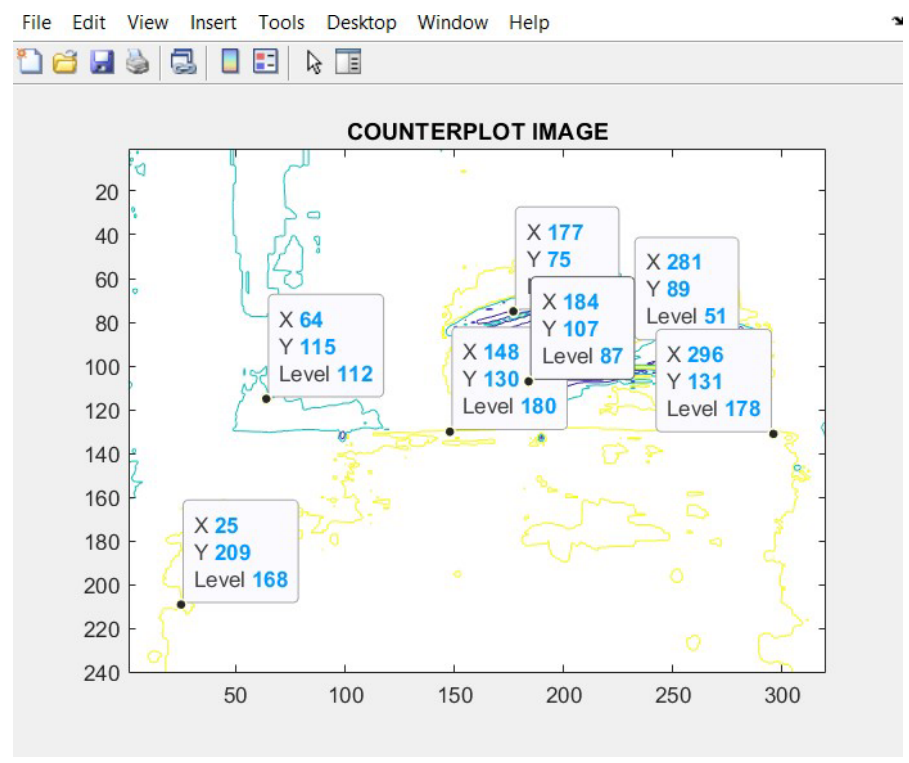

Figure 6. Contour Plot

Table 1. Analysis of Contour plot

\begin{tabular}{|c|c|c|}
\hline $\begin{array}{c}\text { X } \\
\text { Axis }\end{array}$ & $\begin{array}{c}\text { Y } \\
\text { Axis }\end{array}$ & $\begin{array}{c}\text { Level } \\
\text { s }\end{array}$ \\
\hline $\mathbf{2 5}$ & 209 & $\mathbf{1 6 8}$ \\
\hline $\mathbf{6 4}$ & 115 & $\mathbf{1 1 2}$ \\
\hline $\mathbf{1 4 8}$ & 130 & $\mathbf{1 8 0}$ \\
\hline $\mathbf{1 7 7}$ & 75 & $\mathbf{4 4}$ \\
\hline $\mathbf{1 8 4}$ & 107 & $\mathbf{8 7}$ \\
\hline $\mathbf{2 8 1}$ & 89 & 51 \\
\hline $\mathbf{2 9 6}$ & 131 & $\mathbf{1 7 6}$ \\
\hline
\end{tabular}

\subsubsection{Bounding Box}

In object detection, the use a bounding box is to represent the target location. The bounding box is a rectangular box that can be represented in Figure 7 is in the form of the axis coordinates. The origin of the coordinates in the above image is the center of the image. The image represents the identification of the fish within the Bounding Box. It involves the conversion of the images into a collection of regions of pixels that are represented in the form of a mask or a labeled image. By dividing an image into segments, the process can be done by only the important segments of the image rather than processing the entire image.

\subsubsection{Histogram for CLAHE image}

The Figure 8 represents the implementation of the grey scale conversion and the adaptive equalization technique. It states that the total time required for the image to retrieve the output is faster while compared to the blurry input image. 


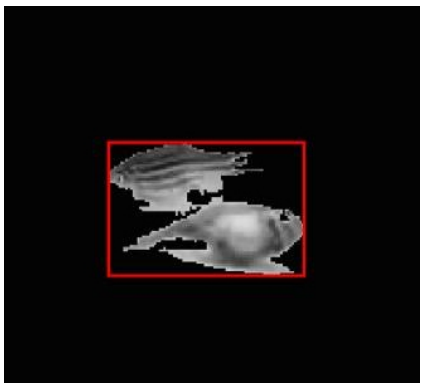

Figure 7. Segmented result

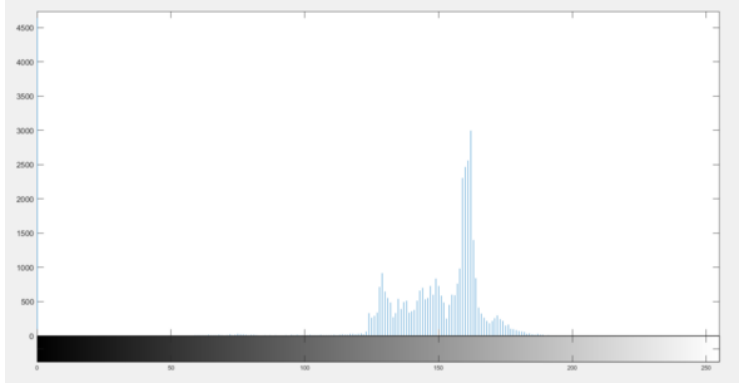

Figure 8. Histogram of the output image

\section{Conclusion}

An efficient approach for similarity calculation between closed contours is proposed. The approach can deal with noisy and distorted shapes and is invariant to translation, scale change and rotation. The proposed dissimilarity measure is based on the geometrical distances between corresponding contour points. The coarse to fine contour-based segmentation refinement is proposed to deal with segmentation challenges in fish measurement. There are three key components for the proposed refinement. It is refined for the segmentation from the coarse level to the fine level to deal with variant fish shapes. The refinement is processed iteratively via affine transforms. Shape models provide rich prior knowledge when estimating the segmentation contours.

\section{References}

[1] Villon S, Chaumont M, Subsol G, Villéger S, Claverie T, Mouillot D. Coral reef fish detection and recognition in underwater videos by supervised machine learning: Comparison between Deep Learning and HOG+ SVM methods. In International Conference on Advanced Concepts for Intelligent Vision Systems 2016 Oct 24 (pp. 160-171). Springer, Cham.

[2] Alippi C, Boracchi G, Camplani R, Roveri M. Detecting external disturbances on the camera lens in wireless multimedia sensor networks. IEEE Transactions on Instrumentation and Measurement. 2010 May 6;59(11):2982-90.

[3] Huang TW, Hwang JN, Rose CS. Chute based automated fish length measurement and water drop detection. In 2016 IEEE International Conference on Acoustics, Speech and Signal Processing (ICASSP) 2016 Mar 20 (pp. 1906-1910). IEEE.

[4] Shelhamer E, Long J, Darrell T. Fully Convolutional Networks for Semantic Segmentation. IEEE Transactions on Pattern Analysis and Machine Intelligence. 1 April 2017 April 1;39(4):640-51.

[5] Chuang MC, Hwang JN, Williams K, Towler R. Automatic fish segmentation via double local thresholding for trawl-based underwater camera systems. In 2011 18th IEEE International Conference on Image Processing 2011 Sep 11 (pp. 3145-3148). IEEE.

[6] Long J, Shelhamer E, Darrell T. Fully convolutional networks for semantic segmentation. In Proceedings of the IEEE conference on computer vision and pattern recognition 2015 (pp. 3431-3440).

[7] Yan T. Cultural changes in coral community based on image segmentation and travel public service in coastal areas. Arabian Journal of Geosciences. 2021 Aug;14(15):1-4.

[8] Wang N, Er MJ, Han M. Generalized single-hidden layer feedforward networks for regression problems. IEEE transactions on neural networks and learning systems. 2014 Jul 15;26(6):1161-76.

[9] Kanchev V, Tonchev K, Boumbarov O. Blurred image regions detection using wavelet-based histograms and SVM. In Proceedings of the 6th IEEE International Conference on Intelligent Data Acquisition and Advanced Computing Systems 2011 Sep 15 (Vol. 1, pp. 457-461). IEEE. 
[10] Mathiassen JR, Misimi E, Bondø M, Veliyulin E, Østvik SO. Trends in application of imaging technologies to inspection of fish and fish products. Trends in Food Science \& Technology. 2011 Jun 1;22(6):25775.

[11] Chuang MC, Hwang JN, Williams K, Towler R. Tracking live fish from low-contrast and low-framerate stereo videos. IEEE Transactions on Circuits and Systems for Video Technology. 2014 Sep 12;25(1):167-79.

[12] Liu R, Li Z, Jia J. Image partial blur detection and classification. In 2008 IEEE conference on computer vision and pattern recognition 2008 Jun 23 (pp. 1-8). IEEE.

[13] Achanta R, Shaji A, Smith K, Lucchi A, Fua P, Süsstrunk S. SLIC superpixels compared to state-ofthe-art superpixel methods. IEEE transactions on pattern analysis and machine intelligence. 2012 May 29;34(11):2274-82.

[14] Chuang MC, Hwang JN, Williams K, Towler R. Automatic fish segmentation via double local thresholding for trawl-based underwater camera systems. In2011 18th IEEE International Conference on Image Processing 2011 Sep 11 (pp. 3145-3148). IEEE.

[15] Ravanbakhsh M, Shortis MR, Shafait F, Mian A, Harvey ES, Seager JW. Automated Fish Detection in Underwater Images Using Shape-Based Level Sets. The Photogrammetric Record. 2015 Mar;30(149):46-62.

[16] Jäger J, Rodner E, Denzler J, Wolff V, Fricke-Neuderth K. Object Proposal Classification for Fish Detection in Underwater Videos. InCLEF (working notes) 2016 (pp. 481-489).

[17] Zivkovic Z, Van Der Heijden F. Efficient adaptive density estimation per image pixel for the task of background subtraction. Pattern recognition letters. 2006 May 1;27(7):773-80.

[18] Mathiassen JR, Misimi E, Bondø M, Veliyulin E, Østvik SO. Trends in application of imaging technologies to inspection of fish and fish products. Trends in Food Science \& Technology. 2011 Jun 1;22(6):25775.

[19] Chuang MC, Hwang JN, Williams K, Towler R. Tracking live fish from low-contrast and low-framerate stereo videos. IEEE Transactions on Circuits and Systems for Video Technology. 2014 Sep $12 ; 25(1): 167-79$. 